\title{
Topological Methods IN Nonlinear Analysis
}

\section{ON EXISTENCE OF PERIODIC SOLUTIONS \\ FOR KEPLER TYPE PROBLEMS}

\author{
Pablo Amster — Julián Haddad \\ Topol. Methods Nonlinear Anal. 48 (2016), 465-476 \\ DOI: $10.12775 /$ TMNA.2016.053
}

Published by the

Juliusz Schauder Center

TORUŃ, 2016

ISSN 1230-3429 
This e-offprint is for personal use only and shall not be self-archived in electronic repositories. If you wish to self-archive your article, you may use the accepted manuscript pre-print version for positioning on your own website, provided that the journal reference to the published version (with DOI and published page numbers) is given. You may further deposit the accepted manuscript pre-print version in any repository, provided it is only made publicly available 12 months after official publication or later and provided acknowledgement is given to the original source of publication and a link is inserted to the published article on the TMNA website. 


\title{
Author's personal copy
}

Topological Methods in Nonlinear Analysis

Volume 48, No. 2, 2016, 465-476

DOI: $10.12775 /$ TMNA.2016.053

(C) 2016 Juliusz Schauder Centre for Nonlinear Studies

Nicolaus Copernicus University

\section{ON EXISTENCE OF PERIODIC SOLUTIONS \\ FOR KEPLER TYPE PROBLEMS}

\author{
PABlo Amster - Julián Haddad
}

\begin{abstract}
We prove existence and multiplicity of periodic motions for the forced 2-body problem under conditions of topological character. In different cases, the lower bounds obtained for the number of solutions are related to the winding number of a curve in the plane and the homology of a space in $\mathbb{R}^{3}$.
\end{abstract}

\section{Introduction}

Let us consider the following periodic singular problem:

$$
\left\{\begin{array}{l}
u^{\prime \prime}(t) \pm \frac{u(t)}{|u(t)|^{q+1}}=\lambda h(t), \\
u(0)=u(1) \\
u^{\prime}(0)=u^{\prime}(1)
\end{array}\right.
$$

for a vector function $u: I:=[0,1] \rightarrow \mathbb{R}^{n}$, where $q \geq 2, \lambda>0$ and $h \in C\left(I, \mathbb{R}^{n}\right)$ with $\bar{h}:=\int_{0}^{1} h(t) d t=0$ and $h(0)=h(1)$. Here, $u$ describes the motion of a particle under a singular central force that can be attractive or repulsive depending on the sign \pm , and an arbitrary perturbation $h$.

The case $n=2$ was studied in [2], where the existence of periodic solutions under a non-degeneracy condition was proven. In more precise terms, a lower

2010 Mathematics Subject Classification. 34B15, 34C25, 70K40.

Key words and phrases. Forced 2-body problem; periodic solutions; multiplicity; averaging method; topological degree.

This work was partially supported by the project UBACyT 20020120100029BA. 


\section{Author's personal copy}

bound of the number of solutions depending purely on a topological property of the second primitive of $h$ was obtained, namely:

THEOREM 1.1. Let $H$ be a periodic function such that $H^{\prime \prime}=-h$ and let $r$ be the number of bounded connected components of $\mathbb{R}^{2} \backslash \operatorname{Im}(H)$. Then for $\lambda$ large enough, problem (1.1) has at least $r$ solutions.

In the present work, this result shall be extended in several directions. In Section 2, we consider the repulsive case. We obtain at least one extra solution from the direct computation of the Leray-Schauder degree over the set of curves that are bounded away from the origin and from infinity. Furthermore, we obtain a lower bound of the number of solutions that depends not only on the number of connected components of $\mathbb{R}^{2} \backslash \operatorname{Im}(H)$ but also on the winding number of $H$ with respect to these components. More specifically, if $r$ is the number of bounded connected components of $\mathbb{R}^{2} \backslash H$ and $H$ has $s$ different winding numbers with respect to these components, then the number of solutions is at least $r+s$. As we shall point out, the number of solutions is generically (i.e. for a 'large' set of functions $h$ ) at least equal to $2 r$.

In Section 3 we give some examples illustrating existence and non-existence of solutions in some particular situations.

In Section 4 we present a version of Theorem 1.1 for higher dimensions. Our proofs make use of some classical results of algebraic topology. The case $n=3$ is treated separately because the homology of open sets with smooth boundary is simple and easy to understand. The role of $r$ in Theorem 1.1 shall be played by the dimension of the first homology group $H_{1}\left(\mathbb{R}^{3} \backslash \operatorname{Im}(H)\right)$ : as we shall see, if this number is positive then the problem has a solution for $\lambda$ large enough. We recall that this case can be also treated by means of knot invariants [6]. The case $n>3$ needs more restrictive hypotheses; however, we are able to guarantee the existence of solutions, provided that $H$ is an embedding. Our results can be extended to the restricted $N$-body problem.

1.1. Preliminaries. Theorem 1.1 was proven in [2] using a result essentially contained in Cronin's book [5] that makes use of the so-called averaging method. We also refer to [7, Section V.3] and the more recent paper [11] for a more detailed account of the method and its history. However, for our purposes it shall be convenient to describe the procedure in a precise way. As before, let $H: S^{1} \rightarrow \mathbb{R}^{n}$ be a periodic second primitive of $-h$ (which is unique up to translations). For convenience we shall assume, without loss of generality, that

$$
\bar{H}:=\int_{0}^{1} H(t) d t=0 .
$$

Let us make the change of variables

$$
u(t)=\lambda(z(t)-H(t))
$$




\section{Author's personal copy}

so equation (1.1) becomes

$$
\left\{\begin{array}{l}
z^{\prime \prime}(t)=\mp \varepsilon \frac{z(t)-H(t)}{|z(t)-H(t)|^{q+1}}, \\
z(0)=z(1) \\
z^{\prime}(0)=z^{\prime}(1),
\end{array}\right.
$$

where

$$
\varepsilon=\lambda^{-(q+1)}
$$

Let $C_{\text {per }}^{2}\left(I, \mathbb{R}^{n}\right)$ denote the set of 1-periodic $C^{2}$ functions and define

$$
\widetilde{C}_{\text {per }}^{i}\left(I, \mathbb{R}^{n}\right)=\left\{u \in C_{\text {per }}^{i}\left(I, \mathbb{R}^{n}\right): \bar{u}=0\right\}
$$

for $i=0,1, \ldots$ and $N u:= \pm u(t) /|u(t)|^{q+1}$. For $\varepsilon>0$, it is well-known (see e.g. [10]) that (1.3) can be re-written as a fixed point operator equation

$$
z=\bar{z}-\overline{N(z-H)}-\varepsilon K[N(z-H)-\overline{N(z-H)}]
$$

where $K$ is the inverse of the linear operator $L u:=u^{\prime \prime}$, regarded as an isomorphism from $\widetilde{C}_{\text {per }}^{2}$ onto $\widetilde{C}_{\text {per }}$.

We shall use the Leray-Schauder degree in order to prove that the solutions of (1.5) for $\varepsilon=0$ can be continued for small values of $\varepsilon$. With this aim, let $\mathcal{A}:=\left\{z \in C_{\text {per }}^{2}\left(I, \mathbb{R}^{n}\right): z \neq H\right\}$ and consider the operator $\Phi_{0}: \mathcal{A} \rightarrow C_{\text {per }}^{2}\left(I, \mathbb{R}^{n}\right)$ given by

$$
\Phi_{0}(z):=z-\bar{z}+\overline{N(z-H)} .
$$

From the definition of the Leray-Schauder degree, it is seen that the degree of $\Phi_{0}$ coincides with the Brouwer degree of its restriction to the finite dimensional space of constant functions, namely the function $F: \mathbb{R}^{n} \backslash \operatorname{Im}(H) \rightarrow \mathbb{R}^{n}$ given by

$$
F(x)=\int_{0}^{1} \frac{x-H(t)}{|x-H(t)|^{q+1}} d t .
$$

This setting was enough to prove Theorem 1.1; however, for the results in the present work a more careful analysis is required. Let us define the continuous functional $\mathcal{G}_{\varepsilon}: \mathcal{A} \rightarrow C_{\text {per }}^{2}\left(I, \mathbb{R}^{n}\right)$ given by

$$
\mathcal{G}_{\varepsilon}(z):=z-\bar{z}+\overline{N(z-H)}+\varepsilon K[N(z-H)-\overline{N(z-H)}] .
$$

Thus, for $\varepsilon>0$, the zeros of $\mathcal{G}_{\varepsilon}$ are exactly the solutions of (1.3). Then, with each open set $D \subseteq \mathbb{R}^{n} \backslash \operatorname{Im}(H)$ we may associate the open set of curves $\mathcal{D}=$ $\left\{z \in C_{\text {per }}^{2}\left(I, \mathbb{R}^{n}\right) / \operatorname{Im}(z) \subset D\right\}$ and hence $\operatorname{deg}\left(\mathcal{G}_{\varepsilon}, \mathcal{D}, 0\right)=\operatorname{deg}(F, D, 0)$ for small $\varepsilon$, provided that (1.3) has no solutions over $\partial \mathcal{D}$.

Thus, it suffices to look for open sets $D$ such that the $\operatorname{deg}(F, D, 0)$ is defined and different from zero. For each of these sets $D$ there exists a solution of (1.3) for $\varepsilon>0$ small. In the situation of Theorem 1.1, if $\Omega_{1}, \ldots, \Omega_{r}$ are the bounded 


\section{Author's personal copy}

connected components of $\mathbb{R}^{2} \backslash \operatorname{Im}(H)$, we may construct open sets $\Omega_{i}^{*} \subset \overline{\Omega_{i}^{*}} \subset \Omega_{i}$ where all the respective degrees are equal to 1.

For convenience, we shall consider another functional $\mathcal{F}$ associated to equation (1.1) and independent of $\lambda$, namely

$$
\mathcal{F}(u):=u-\bar{u}+\overline{N u}+K(N u-\overline{N u}) .
$$

The relation between these two functionals is the following: the function $u$ is a solution of (1.1) if and only if $\mathcal{F}(u)=-\lambda H$, if and only if $\mathcal{G}_{\varepsilon}(u / \lambda+H)=$ $\mathcal{G}_{\varepsilon}(z)=0$, if and only if $z=u / \lambda+H$ is a solution of (1.3). By standard properties of the Leray-Schauder degree, it is clear that

$$
\operatorname{deg}\left(\mathcal{G}_{\mathcal{E}}, \mathcal{D}, 0\right)=\operatorname{deg}(\mathcal{F}, \mathcal{E},-\lambda H)
$$

where $\varepsilon$ and $\lambda$ are related by (1.4) and $\mathcal{D}$ and $\mathcal{E}$ are related by

$$
\mathcal{D}=H+\frac{1}{\lambda} \mathcal{E}
$$

Finally, let us define the function $g: \mathbb{R}^{n} \backslash\{0\} \rightarrow \mathbb{R}$ by $g(u)=1 /|u|^{q-1}$, so $\nabla g(u)=-(q-1) u /|u|^{q+1}$.

\section{The repulsive case}

In this section we shall improve Theorem 1.1 for the repulsive case. In the first place, let us prove the existence of an extra solution by a direct degree argument:

TheOREM 2.1. In the conditions of Theorem 1.1, the repulsive case of problem (1.1) admits at least $r+1$ solutions for $\lambda$ large.

It is worth noticing that Theorem 2.1 shall be improved as well at the end of this section by studying the winding number of $H$ with respect to the connected components of $\mathbb{R}^{2} \backslash \operatorname{Im}(H)$. However, this latter extension cannot be carried out in higher dimensions; thus, for the sake of clarity it shall be convenient to prove both results separately.

We will make use of the following two lemmas, which shall provide us a priori bounds for the solutions. Later on, these bounds will be used also in the proofs of Theorems 4.1 and 4.8 for higher dimensions, so the results will be stated in $\mathbb{R}^{n}$. We remark that nothing of this can be extended to the attractive case.

Lemma 2.2. Given $\lambda_{*}>0$, there exist constants $R, r>0$ such that $r<$ $|u(t)|<R$ for all $t \in I$, for any $u: I \rightarrow \mathbb{R}^{n}$ solution of (1.1) with $\lambda \leq \lambda_{*}$.

Proof. Let us firstly prove that solutions are uniformly bounded away from the origin. Let $u$ be a solution. We define the energy and compute its derivative

$$
E(t)=\frac{1}{2}\left|u^{\prime}(t)\right|^{2}+\frac{1}{(q-1)|u(t)|^{q-1}}, \quad E^{\prime}(t)=\left\langle u^{\prime}, u^{\prime \prime}\right\rangle-\frac{\left\langle u^{\prime}, u\right\rangle}{|u|^{q+1}}=\left\langle u^{\prime}, \lambda h\right\rangle,
$$




\section{Author's personal copy}

$$
\left|E^{\prime}(t)\right| \leq \lambda\left|u^{\prime}(t)\right||h(t)| \leq \lambda\|h\|_{\infty} \sqrt{2 E(t)} \leq C \lambda_{*} \sqrt{E(t)} .
$$

Now multiply equation (1.1) by $u$ and integrate,

$$
\begin{aligned}
& \int_{0}^{1}\left\langle u, u^{\prime \prime}\right\rangle-\int_{0}^{1} \frac{1}{|u|^{q-1}}=\lambda \int_{0}^{1}\langle h, u\rangle, \\
&-\left\|u^{\prime}\right\|_{2}^{2}-\int_{0}^{1} \frac{1}{|u|^{q-1}}=\lambda \int_{0}^{1}\langle h, u\rangle=\lambda \int_{0}^{1}\langle h, u-\bar{u}\rangle, \\
& \frac{1}{2}\left\|u^{\prime}\right\|_{2}^{2}+\int_{0}^{1} \frac{q-2}{q-1} \frac{1}{|u|^{q-1}}+\int_{0}^{1} E=-\lambda \int_{0}^{1}\langle h, u-\bar{u}\rangle, \\
& \int_{0}^{1} E \leq \lambda\left|\int_{0}^{1}\langle h, u-\bar{u}\rangle\right| \leq \lambda\|h\|_{2}\|u-\bar{u}\|_{2}, \\
& \int_{0}^{1} E \leq \lambda_{*} C\left\|u^{\prime}\right\|_{2} \leq \lambda_{*} C \sqrt{\int_{0}^{1} E .}
\end{aligned}
$$

From this inequality, a bound for $E\left(t_{0}\right)$ for some $t_{0}$ is obtained. Using (2.1), we get a bound for $E(t)$ for all $t$, and hence a bound for $1 /|u(t)|^{q-1}$ depending only on $\lambda_{*}$.

Next, let us prove that solutions are uniformly bounded. By contradiction, suppose there exists a sequence $\left\{u_{n}\right\}$ of solutions with $\left\|u_{n}\right\|_{\infty} \rightarrow \infty$. Then $\left\|u_{n}-\overline{u_{n}}\right\|_{\infty} \leq C\left\|u_{n}^{\prime}\right\|_{\infty} \leq C \sqrt{\|E\|_{\infty}} \leq C$ which, in turn, implies that if $n$ is large then the image of $u_{n}$ lies in a half-space. This contradicts the fact that $\overline{g\left(u_{n}\right)}=0$.

Lemma 2.3. Problem (1.1) has no solutions for $\lambda=0$.

Proof. Let $\lambda=0$ and suppose $u$ is a solution. As before, multiply equation (1.1) by $u$ and integrate, then

$$
-\int_{0}^{1}\left|u^{\prime}\right|^{2}=\int_{0}^{1} u^{\prime \prime} u=\int_{0}^{1} \frac{1}{|u|^{q-1}}
$$

a contradiction.

Lemma 2.4. Solutions of (1.1) are also uniformly bounded in $C^{2}\left(I, \mathbb{R}^{n}\right)$.

Proof. We know from Lemma 2.2 that $\|u\|_{\infty}$ and $\left\|u^{\prime}\right\|_{\infty}$ are bounded and $\inf |u|$ is bounded away from 0 . From (1.1), it follows that $\left\|u^{\prime \prime}\right\|_{\infty}$ is bounded as well.

Lemma 2.5. Let $\mathcal{F}: \mathcal{E} \subset C_{\mathrm{per}}^{2}\left(I, \mathbb{R}^{n}\right) \rightarrow C_{\mathrm{per}}^{2}\left(I, \mathbb{R}^{n}\right)$ where $\mathcal{F}$ is the functional associated to (1.1) and

$$
\mathcal{E}=\left\{u \in C^{2}: r<|u|<R,\left\|u^{\prime}\right\|_{\infty}<C,\left\|u^{\prime \prime}\right\|_{\infty}<C\right\}
$$

where $r, R$ and $C$ are the bounds obtained in the preceding lemmas. Then

$$
\operatorname{deg}\left(\mathcal{F}, \mathcal{E},-\lambda_{*} H\right)=\operatorname{deg}\left(\mathcal{G}_{\varepsilon_{*}}, \mathcal{D}, 0\right)=0
$$




\section{Author's personal copy}

where $\varepsilon_{*}$ and $\mathcal{D}$ are defined as in (1.4) and (1.8).

Proof. It follows immediately from the preceding lemmas, identity (1.7), the homotopy invariance and the solution property of the degree.

Using this fact, we are able to obtain an extra solution of (1.1).

Proof of Theorem 2.1. Following the notation and the proof of Theorem 1.1 in [2], set

$$
\mathcal{A}_{k}:=\left\{z \in C_{\mathrm{per}}^{2}\left(I, \mathbb{R}^{2}\right): \operatorname{Im}(z) \subseteq \Omega_{k}^{*},\left\|z^{\prime}\right\|_{\infty}<C,\left\|z^{\prime \prime}\right\|_{\infty}<C\right\}
$$

with $\Omega_{k}^{*} \subseteq \Omega_{k}$ such that $\operatorname{deg}\left(\nabla g, \Omega_{k}^{*}, 0\right)=1$, and take $\mathcal{E}$ as in the previous lemma. We know that, for some $\varepsilon_{*}$ small enough, $\operatorname{deg}\left(\mathcal{G}_{\varepsilon_{*}}, \mathcal{A}_{k}, 0\right)=1$ and the problem has a solution in $\mathcal{A}_{k}$.

By formula $(1.8), \mathcal{D}=\left\{z: r / \lambda_{*}<|z-H|<R / \lambda_{*}\right\}$, so taking $R$ large enough it is seen that $\mathcal{A}_{k} \subseteq \mathcal{D}$ for all $k$. By the previous lemma, $\operatorname{deg}\left(\mathcal{G}_{\varepsilon_{*}}, \mathcal{D}, 0\right)=0$. Defining $\mathcal{B}=\mathcal{D} \backslash \overline{\bigcup_{k=1}^{r} \mathcal{A}_{k}}$, we obtain $\operatorname{deg}\left(\mathcal{G}_{\varepsilon_{*}}, \mathcal{B}, 0\right)=-r$, so there exists at least one more solution in $\mathcal{B}$, which is obviously different from the others.

In the preceding proof, when $r>1$ it is worth observing that, although the degree of $\mathcal{G}_{\varepsilon_{*}}$ is equal to $-r$, we cannot assert the existence of $r$ different extra solutions since we are not able to ensure that they are non-degenerate. But we are still able to distinguish solutions using properties that are invariant under continuation.

We recall the definition of the index of a curve:

Definition 2.6. Let $\gamma: I \rightarrow \mathbb{R}^{2}$ be a continuous curve and let $x \in \mathbb{R}^{2} \backslash \operatorname{Im}(\gamma)$. Let $j(x, \gamma) \in \mathbb{Z}$ be defined as the winding number of $\gamma$ around $x$. The function $x \mapsto j(x, \gamma)$ is constant in each connected component of $\mathbb{R}^{2} \backslash \operatorname{Im}(\gamma)$. Thus, if $\Omega \subseteq \mathbb{R}^{2}$ is one of these components, we define the winding number $j(\Omega, \gamma)$ of $\gamma$ around $\Omega$.

THEOREM 2.7. In the conditions of Theorem 2.1 , let $\Omega_{1}, \ldots, \Omega_{r}$ be the connected components of $\mathbb{R}^{2} \backslash \operatorname{Im}(H)$ and let $s$ be the cardinality of the set $\left\{j\left(\Omega_{k}, H\right)\right.$ : $k=1, \ldots, r\}$. Then the repulsive case of (1.1) admits at least $r+s$ solutions.

Proof. Let $\mathcal{E}$ be defined as before and consider the "winding number function' $J: \mathcal{E} \rightarrow \mathbb{Z}$ defined by $J(x)=j(0, x)$, which determines in $\mathcal{E}$ the connected components $\mathcal{E}_{i}=\{u \in \mathcal{E}: J(x)=i\}$ for $i \in \mathbb{Z}$. Since $\partial \mathcal{E}=\bigcup_{i \in \mathbb{Z}} \partial \mathcal{E}_{i}$, we deduce from Lemmas 2.2 and 2.3 that $\operatorname{deg}\left(\mathcal{F}, \mathcal{E}_{i},-\lambda H\right)=0$ for every $i$. Using again formula (1.8), we obtain the pairwise disjoint decomposition $\mathcal{D}=\bigcup_{i \in \mathbb{Z}} \mathcal{D}_{i}$ and $\operatorname{deg}\left(\mathcal{G}_{\varepsilon_{*}}, \mathcal{D}_{i}, 0\right)=0$. Using the notation of the previous theorem, we have that 


\section{Author's personal copy}

On Existence of Periodic Solutions for Kepler Type Problems

$\mathcal{A}_{k} \subseteq \mathcal{D}_{i(k)}$ where $i(k):=j\left(\Omega_{k}, H\right)$. Thus, taking

$$
\mathcal{B}_{i}=\mathcal{D}_{i} \backslash \overline{\bigcup_{k: i(k)=i} \mathcal{A}_{k}}=\mathcal{D}_{i} \backslash \overline{\bigcup_{k=1}^{r} \mathcal{A}_{k}},
$$

it is seen that $\operatorname{deg}\left(\mathcal{G}_{\varepsilon_{*}}, \mathcal{B}_{i}, 0\right)=-\#\left\{k / j\left(\Omega_{k}, H\right)=i\right\}$. We remark that this degree is equal to 0 , except for the (finitely many) values of $i$ such that $j\left(\Omega_{k}, H\right)=$ $i$ for some $k$. We conclude that there exists, for each of those values of $i$, at least one solution in $\mathcal{B}_{i}$ which clearly does not belong to $\mathcal{A}_{k}$ for any $k$.

As an example, in [2] we considered, using complex notation, $h(t):=e^{i t}+$ $27 e^{3 i t}$. The function $H(t)=e^{i t}+3 e^{3 i t}$ is a parameterization of the epicycloid:

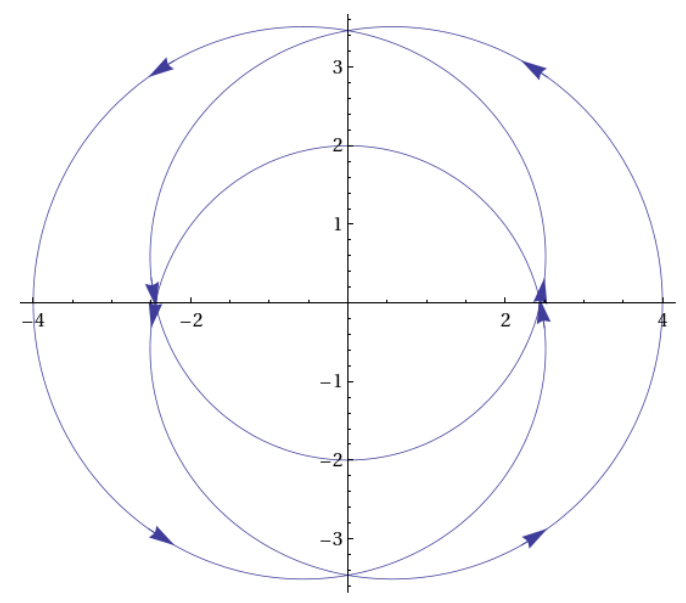

As observed, $\mathbb{R}^{2} \backslash H(\mathbb{R})$ has five bounded connected components with corresponding indices $3,2,2,1,1$. Hence we obtained five periodic solutions. But according to Theorem 2.7, in the repulsive case the number of solutions is at least 8 .

In general, there is no way to guarantee that solutions of a given problem are non-degenerate without knowing them explicitly. But since our functional is smooth, this property can be achieved by arbitrarily small perturbations. It is in some sense a 'generic' property, as stated in the following remark.

Remark 2.8. Using the Sard-Smale Theorem, it can be proven that, for a 'generic' forcing term $h$, the repulsive problem has in fact at least $2 r$ periodic solutions. More specifically, there exists a residual set $\Sigma \in \widetilde{C}_{\text {per }}^{0}$ such that if $\lambda h \in \Sigma$ then all solutions of (1.1) are non-degenerate. Thus, all of them have multiplicity one and depend differentially on $h$. 


\section{Author's personal copy}

\section{Some examples}

Proposition 3.1. For the repulsive case, if $\operatorname{Im}(H)$ is contained in a line then equation (1.3) has no solution for any $\varepsilon>0$.

Proof. Let us take coordinates $(x, y) \in \mathbb{R} \times \mathbb{R}^{n-1}$. We may assume that the $y$ coordinate of $H$ is zero, that is $H(t)=\left(H_{1}(t), 0\right)$.

Let $z(t)=(x(t), y(t))$ be a solution. Multiply $y^{\prime \prime}$ by $y$ and integrate, then

$$
-\int_{0}^{1}\left|y^{\prime}(t)\right|^{2} d t=\int_{0}^{1}\left\langle y(t), y^{\prime \prime}(t)\right\rangle d t=\int_{0}^{1}\left\langle y(t), \frac{\varepsilon y(t)}{|z(t)-H(t)|^{q+1}}\right\rangle d t \geq 0,
$$

so $y \equiv 0$. Now, as $z$ lies in the same line as $H$, we have $H_{1}>x$ or $H_{1}<x$ for all $t$ so either $x^{\prime \prime}$ is positive or negative. This contradicts the fact that $z$ is periodic.

EXAMPLE 3.2. In Theorem 1.1, the existence of bounded connected components of $\mathbb{R}^{2} \backslash \operatorname{Im}(H)$ is not necessary to deduce the existence of solutions.

Indeed, take $H_{l}(t)=e^{i l \sin (t)}$. The curve $H_{l} \subseteq \mathbb{R}^{2}$ is degenerate for $l<\pi$, in the sense that $\mathbb{R}^{2} \backslash \operatorname{Im}\left(H_{l}\right)$ has no bounded connected components.

For $l=\pi$, we may construct an open set $\Omega^{*}$ with $\operatorname{deg}\left(F, \Omega^{*}, 0\right)=1$, where $F$ is the function defined in (1.6). Using the continuity of the Brouwer degree, we deduce that $\operatorname{deg}(F, \Omega, 0)=1$ for some $l<\pi$ close to $\pi$. This provides a periodic solution of (1.1), although $\mathbb{R}^{2} \backslash \operatorname{Im}\left(H_{l}\right)$ has no bounded connected components.

TheOREM 3.3. Assume that $\operatorname{Im}(H)$ is not contained in a line. Then, for $\lambda$ sufficiently large, there exists a solution of the repulsive problem for some re-parameterization of $H$.

Proof. Take $s_{\varepsilon}: I \rightarrow I$ such that $s_{\varepsilon}$ is $C^{1}$ and increasing for $\varepsilon>0$, and $s_{0}$ is piecewise constant, $s_{0}([0, a])=t_{0}, s_{0}((a, 1])=t_{1}$. Define $H_{\varepsilon}(t)=H\left(s_{\varepsilon}(t)\right)$. It is easy to see that $F$ has one non-degenerate zero $x_{0}$ of index -1 . Choosing $a, t_{0}, t_{1}$ appropriately we can ensure that $x_{0}$ is not in $\operatorname{Im}(H)$ (here we use the fact that $\operatorname{Im}(H)$ is not contained in a line) and we take a small neighborhood $U$ of $x_{0}$ not touching $H$. We obtain a zero $x_{1} \in U$ of $F$ for small values of $\varepsilon$ as a non-degenerate zero of index -1 , which is isolated by $U$. This point is continued to a small solution of (1.1) for $\lambda$ large enough and such that

$$
\operatorname{deg}(\mathcal{F}, \mathcal{U},-\lambda H)=-1 \quad \text { where } \mathcal{U}=\left\{x \in C_{\text {per }}^{2} / \operatorname{Im}(x) \subseteq U\right\} .
$$

\section{Higher dimensions}

The proof of Theorem 1.1, as well as its extension for the repulsive case given by Theorem 2.1, can be carried out in dimension $n>2$ without any modification, as long as we can find open sets $\Omega \subset \mathbb{R}^{n} \backslash \operatorname{Im}(H)$ such that the degree over $\Omega$ of the map $F: \mathbb{R}^{n} \backslash \operatorname{Im}(H) \rightarrow \mathbb{R}^{n}$ defined in the introduction is well-defined 


\section{Author's personal copy}

On Existence of Periodic Solutions for Kepler Type Problems

and different from zero. In this section we shall construct open sets with this property. Note, however, that for $n>2$ the set $\mathbb{R}^{n} \backslash \operatorname{Im}(H)$ does not split in distinct connected components. When $n=3$, the role of $r$ shall be played by a different invariant; for $n>3$ we shall assume a more restrictive hypothesis. If $X$ is a topological space, we denote by $H_{*}(X)$ (resp. $\left.\overline{H_{*}}(X)\right)$ the singular homology (resp. reduced homology) groups of $X$, with real coefficients.

4.1. Dimension 3. For convenience, let us define the function

$$
G: \mathbb{R}^{n} \backslash \operatorname{Im}(H) \rightarrow \mathbb{R}
$$

given by

$$
G(x):=\int_{0}^{1} g(x-H(t)) d t=\int_{0}^{1} \frac{1}{|x-H(t)|^{q-1}} d t .
$$

Theorem 4.1. Let $r:=\operatorname{dim}\left(H_{1}\left(\mathbb{R}^{3} \backslash \operatorname{Im}(H)\right)\right)$. Then there exists an open set $\Omega \subseteq \mathbb{R}^{3} \backslash \operatorname{Im}(H)$ such that $\operatorname{deg}(\nabla G, \Omega, 0) \geq r$.

As a consequence of Theorem 4.1 and the proof of Theorem 2.1 we obtain

Corollary 4.2. If $r>0$ then problem (1.1) admits a solution for $\lambda>0$ large enough, and two solutions for the repulsive case.

REMARK 4.3. In the case that $\operatorname{Im}(H)$ is contained in a plane $P$, it is easy to see that $H_{1}\left(\mathbb{R}^{3} \backslash \operatorname{Im}(H)\right) \cong \overline{H_{0}}(P \backslash \operatorname{Im}(H))$. Then $r$ is the number of bounded connected components of $P \backslash \operatorname{Im}(H)$. In this way, we recover Theorem 1.1, although not in its full generality: indeed, the previous corollary allows to ensure only the existence of one solution.

Our proof of Theorem 4.1 will require several lemmas; all of them are stated in $\mathbb{R}^{n}$. The following properties of $G$ are proven in [6, Theorem 8].

Lemma 4.4. The function $G$ is $C^{\infty}$ smooth in $\mathbb{R}^{n} \backslash \operatorname{Im}(H)$. Moreover:

(a) The function $G$ is sub-harmonic for $q>n-1$ and harmonic for $q=n-1$. In particular, it has no local maxima in the interior of the domain of definition. In consequence, if $U \subseteq \mathbb{R}^{n}$ is an open and bounded set such that $H \cap \bar{U}=\emptyset$ then it attains its maximum at the boundary.

(b) If $B$ is a large ball centered at the origin then $-\nabla G$ is homotopic to the identity in $\partial B$ by a homotopy of non-vanishing vector fields.

Now some general lemmas:

Lemma 4.5. For $n \geq 3$ the set $\mathbb{R}^{n} \backslash \operatorname{Im}(H)$ is arcwise-connected.

Proof. It follows as an application of transversality. Indeed, if a smooth curve $\gamma$ joins two points not in $\operatorname{Im}(H)$, it can be homotoped with fixed end-points in $\mathbb{R}^{3}$ to be transversal to $H$. Since the ranks of the differentials of two curves sum at most 2, transversality in this case means $\gamma$ and $H$ are disjoint. 


\section{Author's personal copy}

The following lemma is a particular case of [12, p. 296, Theorem 16] (Alexander duality), dealing only with homology with real coefficients of smooth manifolds.

LEMma 4.6. Let $U$ be an open set in $S^{n}$ with smooth boundary. If $k_{*}$ and $e_{*}$ denote the reduced Betti numbers of $U$ and $S^{n} \backslash U$ respectively, then $k_{i}=e_{n-1-i}$.

For convenience, if $U \subset \mathbb{R}^{n}$ is open and bounded and $\phi: \partial U \rightarrow \mathbb{R}^{n} \backslash\{0\}$ is continuous, we shall use the notation $\operatorname{deg}(\phi, \partial U, 0):=\operatorname{deg}(\widehat{\phi}, U, 0)$ where $\widehat{\phi}: \bar{U} \rightarrow$ $\mathbb{R}^{n}$ is any continuous extension of $\phi$.

Lemma 4.7 (Hopf, see [8, Satz VI]). Let $U$ be an open bounded set in $\mathbb{R}^{n}$ with smooth boundary and let $\bar{n}: \partial U \rightarrow \mathbb{R}^{n}$ be the outward-pointing unit normal vector field. Then $\operatorname{deg}(\bar{n}, \partial U, 0)=\chi(U)$, where $\chi$ denotes the Euler characteristic.

Proof of TheOrem 4.1. We will construct an open set $U$ with the following properties:

- $\partial U$ is smooth.

- $U \supseteq \operatorname{Im}(H)$.

- $G$ is constant and $\nabla G \neq 0$ in $\partial U$.

- $\chi(U) \leq 1-r$ where $r:=\operatorname{dim}\left(H^{1}\left(\mathbb{R}^{3} \backslash \operatorname{Im}(H)\right)\right)$.

Once we have this set $U$, we may notice that $-\nabla G$ is orthogonal to $\partial U$, so clearly $\operatorname{deg}(-\nabla G, \partial U, 0)=\operatorname{deg}(\bar{n}, \partial U, 0)$ where $\bar{n}$ is the outward-pointing unit normal vector field. From Lemma 4.7 we deduce that $\operatorname{deg}(-\nabla G, \partial U, 0)=\chi(U) \leq$ $1-r$. Next, take a large ball $B$ given by Lemma $4.4(\mathrm{~b})$ and observe that $\operatorname{deg}(-\nabla G, \partial B, 0)=1$. Finally, since $\operatorname{Im}(H) \subseteq U$, it follows that $G$ is well-defined in $\Omega=B \backslash U$ and $\operatorname{deg}(-\nabla G, \Omega, 0) \geq r$.

Now we may construct $U$ as follows. For convenience, we shall regard $\mathbb{R}^{3}$ as embedded in $S^{3}$, and call $N \in S^{3}$ the north pole. We remark that the function $G$ can be extended continuously to $N$ by setting $G(N)=0$.

From the assumptions, there exist smooth curves $\gamma_{1} \ldots \gamma_{r} \in \mathbb{R}^{3} \backslash \operatorname{Im}(H)$ that form a basis of $H_{1}\left(\mathbb{R}^{3} \backslash \operatorname{Im}(H)\right)$. By Lemma 4.5, each $\gamma_{i}$ is connected to $N$ by another curve $\delta_{i}$. Since $G$ is continuous in $\bigcup \gamma_{i} \cup \delta_{i}$, it is bounded there by a number $\alpha_{0}$. By Sard's lemma, there exists a regular value $\alpha>\alpha_{0}$.

Next, take $V$ the connected component of $\{G<\alpha\}$ that contains $N$ and let $U=\bar{V}^{c}$. $U$ and $V$ are manifolds with common boundary in $S^{3}$. Moreover, $U$ has a finite number of connected components that are disjoint manifolds with boundary. Obviously $\operatorname{Im}(H) \subseteq U$.

We claim that $U$ is in fact connected. Indeed, let $U^{\prime}$ be a connected component of $U$. Then $U^{\prime} \cap \operatorname{Im}(H) \neq \emptyset$ : otherwise, from Lemma 4.4 (a) we deduce that $\left.G\right|_{U^{\prime}}$ attains its maximum at some $x_{0} \in \partial U^{\prime} \subseteq \partial U$, so $G\left(x_{0}\right)=\alpha$. But also $G \leq \alpha$ in $V$, so $x_{0}$ is a local maximum of $G$ in $\mathbb{R}^{3} \backslash \operatorname{Im}(H)$, a contradiction since 


\section{Author's personal copy}

$\alpha$ is a regular value. Moreover, $\operatorname{Im}(H) \subseteq U$ is connected, so we conclude that $U$ is connected. Since $G=\alpha$ in $\partial U$ and $G<\alpha$ in $\bigcup \gamma_{i} \cup \delta_{i}$, it follows that $\gamma_{i} \subseteq V$ and that $U$ is bounded.

Now observe that the inclusion $H_{1}(V) \rightarrow H_{1}\left(\mathbb{R}^{3} \backslash \operatorname{Im}(H)\right)$ induces a homomorphism, which sends $\left[\gamma_{i}\right]_{H_{1}(V)}$ to the generators, so it is onto. Using the notation of Lemma 4.6, we conclude that $e_{1}=\operatorname{dim}\left(H_{1}(V)\right) \geq r$.

Using the Alexander duality (Lemma 4.6) and the fact that $V$ is connected it is seen that $k_{2}=e_{0}=0$. Also, $k_{0}=0$ because $U$ is connected, and $k_{1}=e_{1} \geq r$. The Euler characteristic of $U$ is $\chi(U)=1+k_{0}-k_{1}+k_{2} \leq 1-r$, and this completes the proof.

4.2. Dimension $n>3$. In this section we shall prove, for $n>3$, the existence of a set $\Omega$ as in the proof of Theorem 4.1.

THEOREM 4.8. If $H$ is an embedding then there exists an open set $\Omega$ such that $\operatorname{deg}(-\nabla G, \Omega, 0)=1$. Thus, equation (1.1) has a periodic solution for $\lambda$ large enough.

The following lemma is proven in $[6$, p. 62$]$ for $n=3, q=2$ and the proof carries out without further modifications.

LEMMA 4.9. There is an arbitrarily small tubular neighbourhood $U$ of $\operatorname{Im}(H)$ which is transversal to the vector field $\nabla G$.

Proof of Theorem 4.8. From Lemma 4.7, $\operatorname{deg}(-\nabla G, \partial U, 0)=\chi(U)=$ $\chi\left(S^{1}\right)=0$, then taking $\Omega=B \backslash U$, we have that $\operatorname{deg}(-\nabla G, \Omega, 0)=1$.

REMARK 4.10. As the set of embeddings of $S^{1}$ in $\mathbb{R}^{n}$ for $n \geq 3$ is open in the $C^{1}$ topology and dense in the $C^{\infty}$ topology, Theorem 4.8 is a result about the generic situation.

REMARK 4.11. The preceding results can be generalized to the restricted $N$-body problem, namely

$$
\left\{\begin{array}{l}
z^{\prime \prime}(t)=\mp \varepsilon \sum_{i=1}^{N-1} \frac{z(t)-H_{i}(t)}{\left|z(t)-H_{i}(t)\right|^{q+1}}, \\
z(0)=z(1), \\
z^{\prime}(0)=z^{\prime}(1),
\end{array}\right.
$$

for $z: I \rightarrow \mathbb{R}^{3}$, where $H_{i}: I \rightarrow \mathbb{R}^{3}$ are arbitrary periodic functions. This equation describes the motion of a particle $z$ under the force of gravitational attraction of $N-1$ bodies moving along large periodic trajectories $H_{i}(t)$. Observe that $N$ stands for the number of bodies and not for the dimension, which is now 3 .

Then taking $K=\bigcup_{i=1}^{N-1} \operatorname{Im}\left(H_{i}\right)$ it can be seen using the technique presented before for equation (1.3), that if $r=\operatorname{dim}\left(H_{1}\left(\mathbb{R}^{3} \backslash K\right)\right) \geq N-1$ then for $\varepsilon>0$ 


\section{Author's personal copy}

small enough, problem (4.1) has at least one periodic solution, and generically at least $r-N+2$ distinct solutions.

Acknowledgements. The authors thank the anonymous referee for careful reading of the manuscript and helpful remarks.

\section{REFERENCES}

[1] R. Abraham, J.E. Marsden and T.S. Ratiu, Manifolds, Tensor Analysis and Applications, Applied Mathematical Sciences 75, Springer, 1989.

[2] P. Amster, J. Haddad, R. Ortega and A. J. Ureña, Periodic motions in forced problems of Kepler type, Nonlinear Differential Equations Appl. 18 (2011), 649-657.

[3] P. Amster and M. Maurette, Periodic solutions of systems with singularities of repulsive type, Adv. Nonlinear Stud. 11 (2011), 201-220.

[4] A. Banyaga and D. Hurtubise, Lectures on Morse Homology, Kluwer Texts in the Mathematical Sciences. Vol. 29, Springer, 2004.

[5] J. Cronin, Fixed Points and Topological Degree in Nonlinear Analysis, Math. Surveys No. 11, Amer. Math. Soc. Providence R.I., 1964.

[6] J. Haddad and P. Amster, Critical point theory in knot complements, Differential Geom. Appl. 36 (2014), 56-65.

[7] J. Hale, Ordinary Differential Equations, Krieger Publishing Company, 1969.

[8] H. Hopf, Vektorfelder in n-dimensionalen Mannigfaltigkeiten, Math. Ann. 96 (1926/1927), $225-250$.

[9] P. Martinez-Amores, J. Mawhin, R. Ortega and M. Willem, Generic results for the existence of nondegenerate periodic solutions of some differential systems with periodic nonlinearities, J. Differential Equations 91 (1991), 138-148.

[10] J. Mawhin, Topological degree methods in nonlinear boundary value problems, Regional Conf. Ser. Math. 40, Amer. Math. Soc., Providence, RI, 1979.

[11] J. Mawhin, Periodic solutions in the goldensSixties: the birth of a Continuation Theorem, Ten Mathematical Essays on Approximation in Analysis and Topology (J. Ferrera, J. LópezGómez, F.R. Ruiz del Portal, eds.), Elsevier 2005, 199-214.

[12] E.H. Spanier, Algebraic Topology, Springer, 1994.

Pablo Amster

Departamento de Matemática

Facultad de Ciencias Exactas y Naturales

Universidad de Buenos Aires and IMAS-CONICET

Ciudad Universitaria Pab. I

1428 Buenos Aires, ARGENTINA

E-mail address: pamster@dm.uba.ar

JULIÁN HADDAD

Departamento de Matemática, ICEx

Universidade Federal de Minas Gerais

30.123-970, Belo Horizonte, BRASIL

E-mail address: julianhaddad@ufmg.br

TMNA : Volume $48-2016-\mathrm{N}^{\mathrm{O}} 2$ 